\title{
Imagerie globale de la Terre par les ondes sismiques
}

\section{Barbara Romanowicz (barbara@ipgp.fr)}

Professeure au Collège de France et membre de l'Académie des sciences Institut de Physique du Globe de Paris, 1 rue Jussieu, 75238 Paris Cedex 05

\section{L'imagerie du manteau de la Terre}

est motivée par certaines questions

\section{fondamentales en géophysique,}

\section{telle la compréhension}

des processus présents et passés

\section{de dynamique interne à l'origine}

de la tectonique des plaques.

Depuis une vingtaine d'années,

l'étude de l'intérieur de la Terre

connaît une véritable révolution,

notamment grâce au développement

de techniques toujours plus

performantes de tomographie

sismique et à la puissance

de calcul rendue possible

par l'informatique.

À l'heure actuelle, il est possible

de calculer de manière précise

le champ des ondes sismiques

à travers des structures 3D

arbitrairement complexes,

en géométrie sphérique et pour

des durées suffisantes, par des

méthodes de calcul numérique,

telle la méthode des « éléments

spectraux ».

\section{Imagerie statique de l'intérieur de la Terre}

Avant que les techniques d'imagerie sismique ne voient le jour, la structure de l'intérieur de la Terre a donné lieu à de nombreuses spéculations, comme dans le célèbre roman de Jules Verne, Voyage au centre de la Terre (1864). Au début du $19^{\mathrm{e}}$ siècle, les progrès sont minces puisque, par exemple, Edmond Halley explique la présence du champ magnétique terrestre en imaginant que la Terre contient une cavité remplie d'eau.

C'est une évidence, la structure interne de la Terre n'est pas directement accessible (le forage le plus profond n'atteint pas $13 \mathrm{~km}$, soit un tiers de l'épaisseur moyenne de la croûte continentale). Pour connaître et comprendre notre planète, nous devons donc réaliser des mesures à la surface et par satellite et, en parallèle, faire des observations et des modélisations en laboratoire. Nous avons toutefois de la chance : notre planète est constituée, en moyenne, de couches concentriques, ce qui simplifie le problème.

La sismologie globale, une des techniques les plus puissantes pour étudier l'intérieur de la Terre, a vu le jour en 1889 lorsque Ernst von Rebeur-Paschwitz observe simultanément à Postdam et à Wilhelmshaven une perturbation sur des pendules construits pour étudier les marées terrestres. Quelques jours plus tard, il apprend qu'il y a eu un séisme au Japon, à la même date. Il rapproche les deux événements : des ondes, engendrées par ce séisme, ont été perçues par les pendules, après propagation à travers la Terre sur de très grandes distances.
L'imagerie sismique vise à détecter à l'aide de capteurs de vitesse ou d'accélération, les sismomètres ${ }^{(a)}$, dont la sensibilité atteint le micron, les mouvements du sol induits par les ondes émises par les tremblements de terre. Un sismogramme (fig. 1a) enregistre en fonction du temps l'énergie dégagée par le séisme sous la forme de plusieurs types d'ondes élastiques, se propageant soit à travers l'intérieur de la Terre (" ondes de volume ") soit le long de la surface (" ondes de surface »). Il y a deux types d'ondes de volume : les ondes de compression (" ondes $\mathrm{P}$ ») et les ondes de cisaillement (" ondes S ). Leurs propriétés différentes permettent d'obtenir des renseignements sur les structures internes de la planète. Les ondes de volume interagissent avec les différentes discontinuités de structure : le manteau solide, le noyau liquide et la graine solide. Elles se réfractent, se réfléchissent, changent de nature. Les ondes de cisaillement ne se propagent pas dans les fluides. Ainsi, la présence d'un noyau de type fluide a été confirmée en 1906 par Oldham par l'existence d'une "zone d'ombre ", dans laquelle aucune station sismique n'enregistre d'ondes de cisaillement provenant d'un séisme (fig. 1b). Il a estimé le rayon de ce noyau à 3500 km, ce qui est proche de la valeur actuellement admise (3485 km).

Il y a aussi une «zone d'ombre " pour les ondes de compression due à la réfraction à la limite noyau-manteau, qui les fait plonger plus profondément dans le noyau au-delà d'un angle d'incidence critique. C'est ainsi que Inge Lehmann a découvert la graine en 1936 : elle a observé des ondes qui n'étaient pas prévues dans le cas d'un noyau 


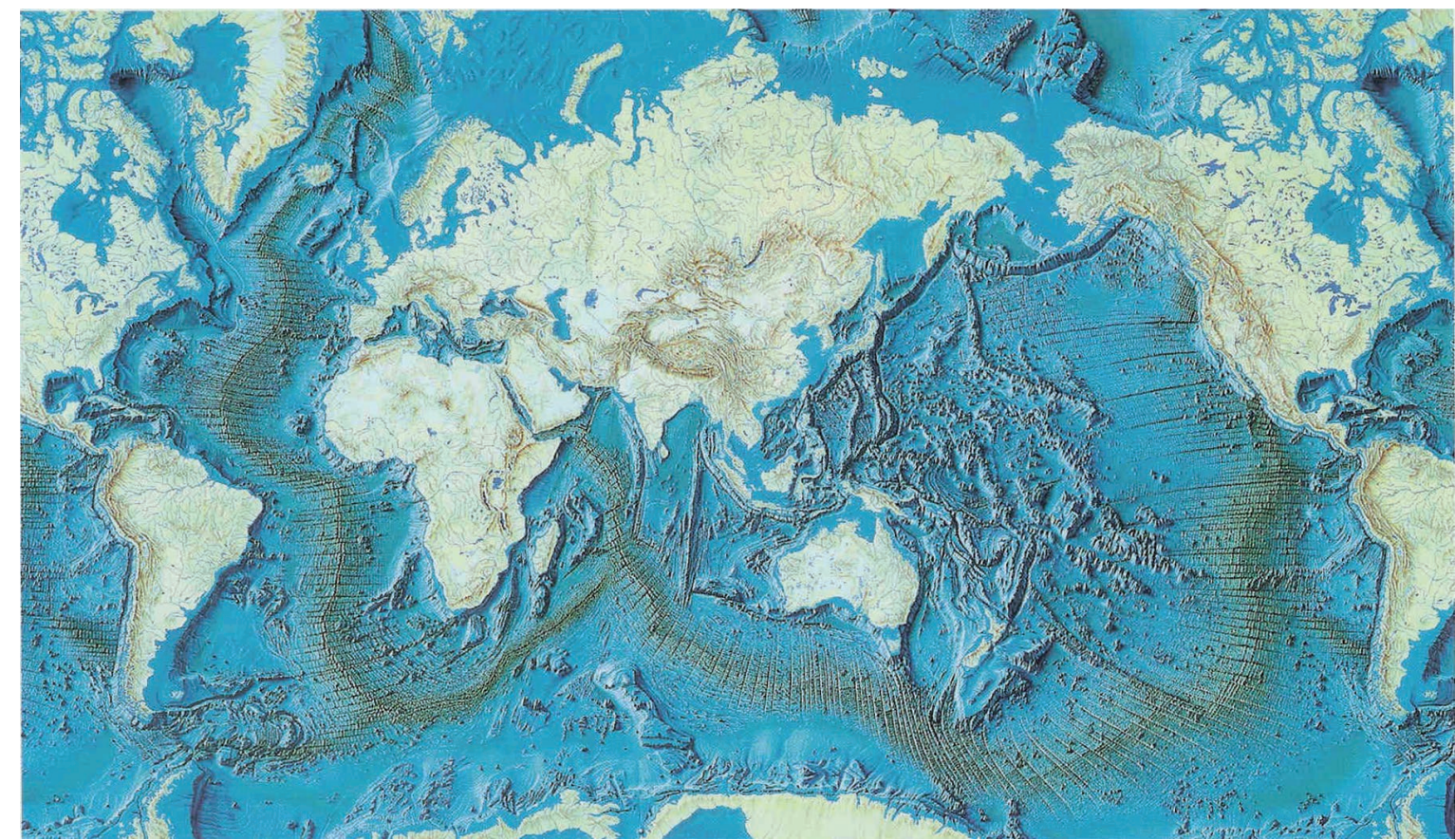

Carte des reliefs mondiaux. La topographie du fond de la mer s'explique par la tectonique des plaques. On note les dorsales qui parcourent le fond des océans sur plus de $60000 \mathrm{~km}$ de long. (Source : X. Le Pichon et al., www.svt-monde.org/spip.php?article44).

fluide homogène et les a attribuées à la présence d'un changement de structure vers le centre de la Terre.

La majeure partie de l'énergie engendrée par un séisme se propage sous la forme d'ondes de surface de longue période (> 30 s). Selon la magnitude du séisme, celles-ci peuvent faire le tour de la Terre de multiples fois avant d'être complètement atténuées. On peut donc suivre l'arrivée de ces trains d'ondes pendant de nombreuses heures. L'énergie diminue au cours du temps avec deux effets : la dispersion géométrique et les pertes d'énergie liées au fait que la Terre n'est pas tout à fait un matériau élastique sans perte.

Si on réalise un enregistrement très long pour un fort tremblement de terre, on mesure sur le spectre de fréquences des « pics " qui sont les modes propres de vibration de la Terre. Leurs fréquences sont caractéristiques de la structure interne de notre planète. Si la Terre était vraiment sphérique, les "pics " seraient des "pics simples »; en fait, la rotation de la Terre et la présence d'hétérogénéités non sphériques provoquent la séparation de ces pics en plusieurs pics de fréquences légèrement différentes, déterminées par ces hétérogénéités.
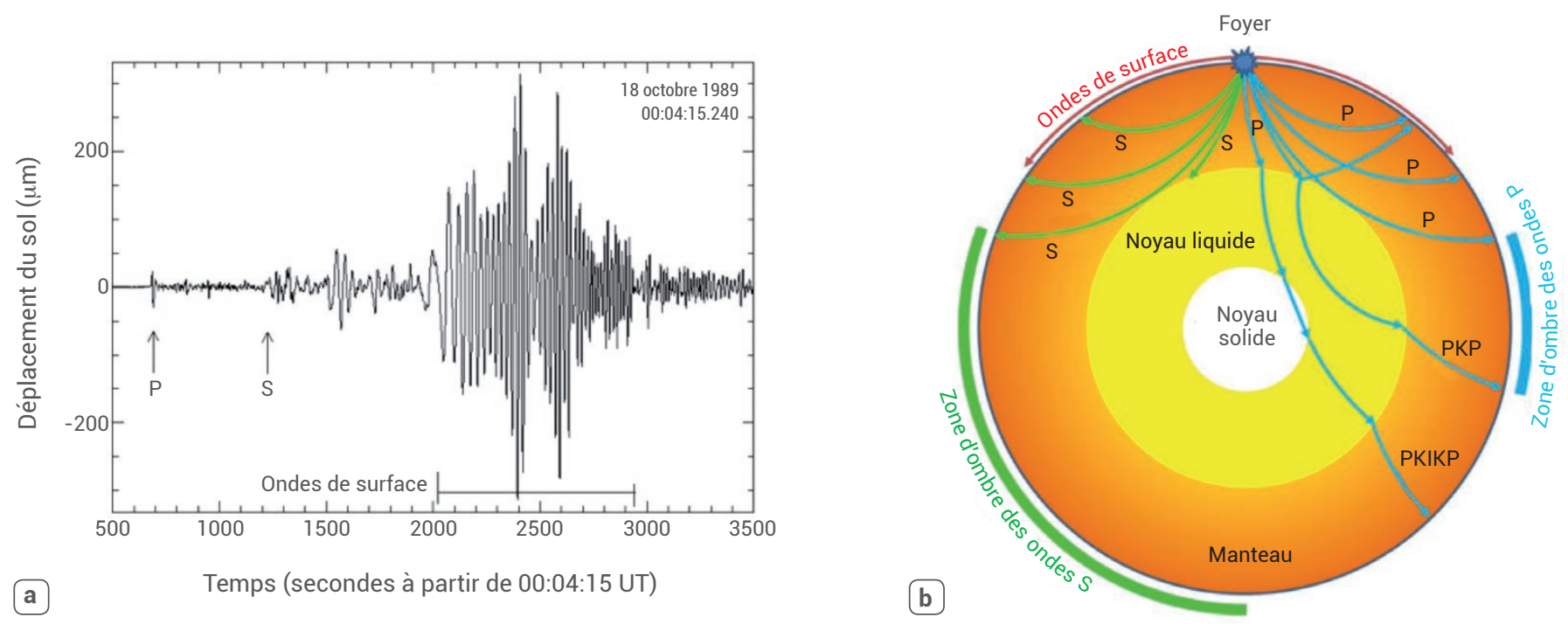

1. Ondes sismiques.

(a) Exemple de sismogramme (séisme de Loma Prieta, dans la baie de San Francisco en Californie, le 17 octobre 1989, observé en Finlande). (Source : Princeton earth physics project).

(b) Différents types d'ondes sismiques. P et $\mathrm{S}$ : ondes de volume du manteau, compressives (P) ou de cisaillement (S). PKP : ondes de compression qui traversent le noyau liquide. PKIKP : ondes de compression qui traversent le noyau liquide et le noyau solide (la graine). Les zones d'ombre pour les ondes $\mathrm{S}$ et $\mathrm{P}$, dues à la présence du noyau liquide, sont indiquées. 
$>>$

$\mathrm{Au}$ milieu du $20^{\mathrm{e}}$ siècle, les données sismologiques nous ont informé sur les propriétés élastiques moyennes : densité et vitesses des ondes de compression et de cisaillement en fonction de la profondeur (fig. 2). L'apport de la physique des matériaux a permis de connaitre la composition moyenne des différentes couches : le manteau principalement constitué de silicates, le noyau externe liquide très riche en fer (avec 10\% d'éléments légers) et la graine solide encore plus concentrée en fer. Dans le manteau supérieur, plusieurs discontinuités de structure globales ont été identifiées comme étant dues aux transformations de phase des silicates en fonction de la pression et de la température. Une nouvelle transformation de phase, découverte récemment (en 2004) dans la pérovskite de magnésium $\left(\mathrm{MgSiO}_{4}\right.$, désormais appelée bridgmanite), composant principal du manteau inférieur, pourrait être associée à une discontinuité sismique observée dans certains endroits autour du globe, à environ 200-300 km au-dessus de la limite noyau-manteau.

\section{Comportement dynamique de l'intérieur de la Terre}

La structure moyenne de la Terre (en "pelures d'oignon ") nous en donne une image statique, mais ne nous renseigne pas sur les processus dynamiques à l'origine des mouvements des plaques tectoniques. En effet, depuis la fin des années 1960, on sait que la Terre est couverte d'une vingtaine de plaques lithosphériques, d'une épaisseur moyenne de $40 \mathrm{~km}$, qui se déplacent les unes par rapport aux autres à la surface. Les déformations sont concentrées à leurs frontières (fig. 3). Le long de zones de divergence (appelées dorsales ou rides), au milieu des océans, une nouvelle croûte se forme en permanence et repousse les plaques, ce qui les fait s'écarter (fig. 3a) ; dans les zones de subduction, les plaques plongent dans le manteau (fig. 3b); il y a aussi des zones, comme la faille de San Andreas en Californie, où les plaques coulissent l'une le long de l'autre (fig. 3c). La théorie de la tectonique des plaques explique ainsi un grand nombre d'observations de surface.

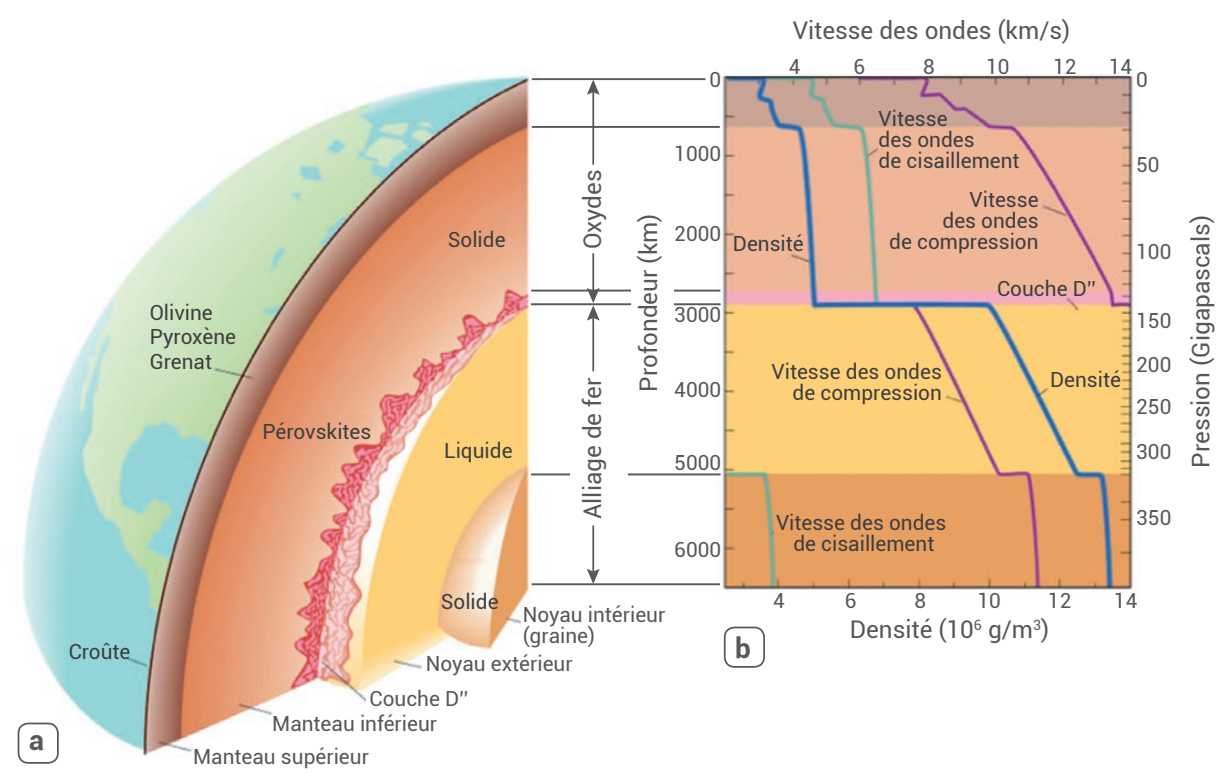

2. Propagation des ondes sismiques à l'intérieur de la Terre.

(a) Modèle de l'intérieur de la Terre. La croûte et le manteau sont formés d'oxydes cristallins, tels que l'olivine, le pyroxène et le grenat dans le manteau supérieur, et des silicates de structure pérovskite dans le manteau inférieur. Le noyau est un alliage de fer, liquide à l'extérieur et solide au centre.

(b) Variation de la densité (courbe bleue) et de la vitesse des ondes élastiques de compression (courbes violettes) et de cisaillement (courbes vertes) avec la profondeur dans le modèle de référence PREM (Preliminary Reference Earth Model, Dziewonski et Anderson, 1981). Dans le manteau, les vitesses des ondes augmentent avec la profondeur, avec des discontinuités à 400 et $600 \mathrm{~km}$ de profondeur. Les discontinuités noyau-manteau et graine-noyau liquide sont bien visibles, avec une vitesse de cisaillement nulle dans le noyau liquide, et à nouveau non nulle dans la graine solide.

(Source : R. Jeanloz et T. Lay, "The Core-Mantle Boundary", Scientific American, 268, n5 (1993) 48-55).
Alfred Wegener a proposé la théorie de la "dérive des continents " dès le début du $20^{\mathrm{e}}$ siècle (1915). Mais, pendant longtemps, cette théorie n'a pas été acceptée, faute de comprendre l'origine des forces en jeu. On sait maintenant que le manteau terrestre, solide aux échelles de temps courts, se comporte comme un matériau visqueux à long terme et rend possible l'écoulement de matière par convection. Cette convection est alimentée par le refroidissement séculaire de la Terre et la production de chaleur dans le manteau terrestre par radioactivité. Les courants de convection dans le manteau terrestre ont des vitesses de quelques $\mathrm{cm}$ par an, du moins près de la surface. On peut le constater en mesurant le déplacement relatif des plaques, mues par des courants ascendants qui font monter le magma issu du manteau supérieur, formant la nouvelle croûte dans les zones de dorsales océaniques, ainsi que des courants descendants qui entrainent les plaques dans le manteau dans les "zones de subduction".

Ces idées simples expliquent une très grande partie des observations de surface, mais pas toutes. Par exemple, les variations du flux de chaleur à la surface et de la topographie des fonds marins en fonction de la distance à la dorsale (c'est-à-dire de l'âge de la plaque) ne sont pas celles attendues. Y a-t-il des sources de chaleur sous le milieu des plaques autres que celles qui sont responsables de la circulation principale définie par les rides et les zones de subduction ? Deux hypothèses ont été proposées :

- Présence d'une convection secondaire ? Lors d'expériences de laboratoire dans un fluide en convection - chauffé par le bas, refroidi par le haut - et recouvert d'une plaque mobile, il se forme des rouleaux de convection secondaire, alignés parallèlement au mouvement de la plaque : les rouleaux de Richter. Ils n'ont pas été observés de manière convaincante jusqu'à présent dans le cas de la Terre.

- Présence de volcanisme intra-plaque ? La tectonique des plaques explique le volcanisme en bordure de plaque. Par contre, elle ne permet pas de comprendre la présence des chaines de volcans " de points chauds " au milieu de l'océan Pacifique, alignés dans la direction du déplacement de la plaque et dont l'âge augmente avec la distance à la dorsale, comme à Hawaï. Tuzo Wilson a proposé en 1963 l'existence de "panaches volcaniques ": ces volcans correspondraient 
a

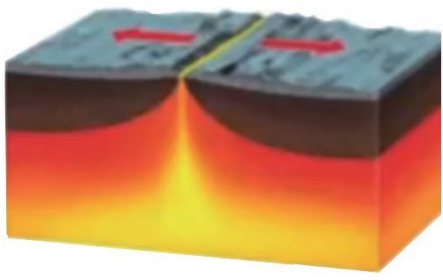

b

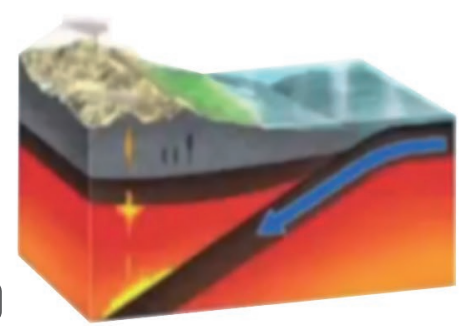

c

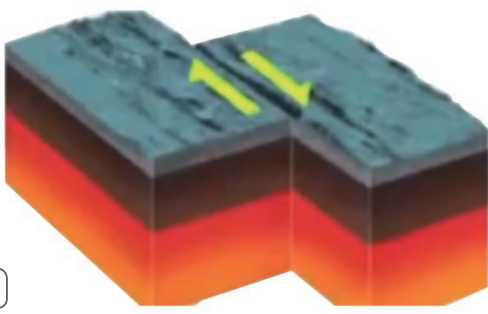

3. Manifestations de la tectonique des plaques en surface.

(a) Formation des plaques dans les dorsales, là où les plaques s'écartent l'une de l'autre.

(b) Subduction d'une plaque sous une autre, là où elles convergent (avec volcanisme associé).

(c) Les deux plaques coulissent l'une par rapport à l'autre, comme dans le cas de la Californie le long de la faille de San Andreas.

à l'arrivée à la surface de la Terre de panaches de matière chaude, dont la racine serait fixée quelque part dans le manteau profond, au niveau d'une couche limite pour la convection. Jason Morgan suggéra en 1972 que les racines des panaches se trouveraient à la limite entre le manteau et le noyau. Nos travaux sont en accord avec cette hypothèse. Des panaches individuels commencent à être discernés dans notre dernier modèle du manteau profond. Ils sont beaucoup plus gros que les panaches classiques obtenus en laboratoire et par les calculs numériques de convection dans des fluides chauffés par le bas. Nous obtenons maintenant des structures de l'ordre de $1000 \mathrm{~km}$ de diamètre. Étant donné la résolution latérale limitée des modèles tomographiques, en réalité leur diamètre pourrait être plus faible, de l'ordre d'un facteur deux, mais il ne serait pas possible de les voir avec nos techniques actuelles si leur diamètre était cinq fois plus petit, comme l'exigent les modèles basés sur une convection d'origine purement thermique. Si leur taille est confirmée, cela démontrerait que ces panaches n'ont pas uniquement une origine thermique et/ou que la rhéologie du manteau profond, telle qu'on la conçoit, est à revoir. Une couche de composition différente à la base du manteau pourrait alimenter ces panaches et fixer leur position pendant des centaines de millions d'années.

\section{Que voit-on à l'intérieur de la Terre par les méthodes tomographiques?}

Pour comprendre et décrire la dynamique interne de notre planète, il est nécessaire d'aller au-delà de la description en couches concentriques de symétrie sphérique, et de cartographier les variations latérales de température et de composition. Pour cela, on fait appel à la tomographie sismique, une imagerie qui repose sur le même principe que l'imagerie médicale, et qui utilise les enregistrements par un réseau de détecteurs des ondes émises par les tremblements de terre pour cartographier en trois dimensions la structure interne du manteau. On part d'un modèle de référence, par exemple un modèle de structure à couches concentriques sphériques homogènes. Les temps de propagation des ondes sont mesurés et comparés à ceux du modèle de référence. Les différences sont expliquées par des écarts de vitesses élastiques, que l'on peut localiser à l'intérieur de la Terre avec plus ou moins de précision. Ces variations de propriétés élastiques sont ensuite interprétées en termes de variations de température (la vitesse de propagation des ondes dans un solide décrôit quand la température augmente) et de composition ; mais, au premier ordre, on peut en général négliger l'effet des variations de composition.

La difficulté de la tomographie sismique vient du fait que les seules sources naturelles d'énergie suffisante sont les tremblements de terre, essentiellement distribués à la limite des plaques. De plus, la distribution des capteurs sismiques n'est pas non plus uniforme autour du globe, car il est encore de nos jours difficile et très coûteux de placer des capteurs de haute qualité au fond des océans, qui pourtant couvrent les deux tiers de la surface de la Terre.
Pendant les trente dernières années, l'instrumentation a fait des progrès importants avec la construction de sismomètres très sensibles, de très large bande passante, qui permettent d'enregistrer toutes les fréquences utiles pour la sismologie globale. On représente la structure interne de la Terre, liée directement à sa dynamique, par des cartes de variations des vitesses sismiques à une profondeur donnée, repérées par rapport à la moyenne globale à cette profondeur (fig. 4). On peut aussi représenter des coupes verticales suivant des profils d'intérêt particulier.

À $100 \mathrm{~km}$ de profondeur (fig. 4a), dans le manteau supérieur, la structure élastique reflète la tectonique des plaques : on observe des régions chaudes (vitesses des ondes de cisaillement plus lentes que la moyenne) le long des dorsales océaniques, le refroidissement progressif de la plaque dans le Pacifique avec l'âge (quand on s'éloigne des dorsales), enfin des régions froides et épaisses au centre des continents - les "cratons ", vieux de plusieurs milliards d'années. Derrière les zones de subduction du Pacifique, des régions chaudes correspondent à des zones d'extension associées au volcanisme "d'arrière-arc ${ }^{(b)}$ ". La carte des reliefs mondiaux de la page 5 montre la topographie des fonds marins ; on peut noter la bonne corrélation entre la position des dorsales médio-océaniques et les régions de vitesse lente (donc plus chaudes) observées sur la figure 4a.

À $600 \mathrm{~km}$ de profondeur (fig. 4b), l'image est très différente ; les seules traces de la tectonique de surface qui subsistent sont des bandes de vitesse des ondes plus rapide que la moyenne dans les zones de subduction de l'ouest du Pacifique et en Amérique du Sud, qui expriment la présence des plaques froides qui replongent dans le manteau.

À 1000 km (fig. 4c), ce qui correspond à environ un tiers de la profondeur du manteau (haut du manteau inférieur), il ne reste des régions froides que dans quelques zones de subduction. La couche de transition entre le manteau supérieur et le manteau inférieur, où la viscosité augmente considérablement (d'un facteur de l'ordre de 30), serait un frein au plongement des plaques au fond du manteau.

À $2800 \mathrm{~km}$ de profondeur (fig. 4d), $100 \mathrm{~km}$ au-dessus de la limite noyaumanteau, la structure a changé complètement avec, maintenant, des structures de 


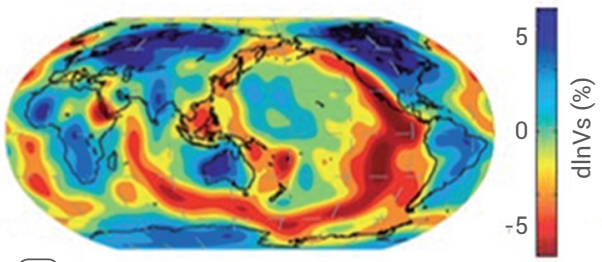

a

100 km

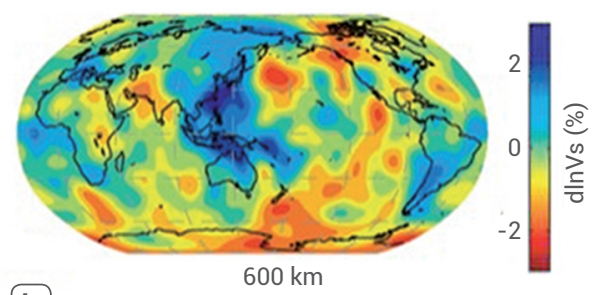

b
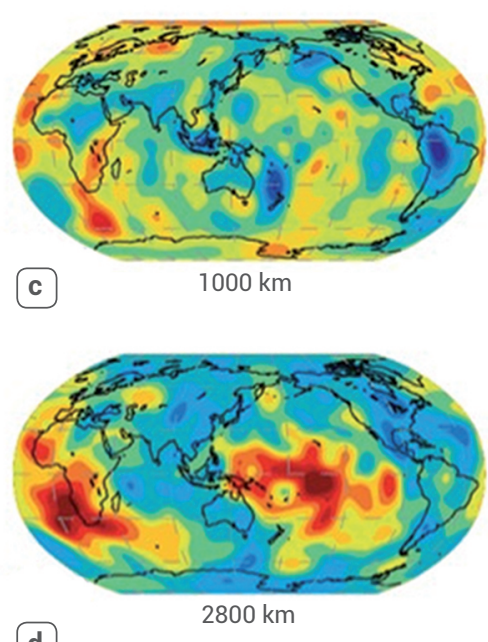

d
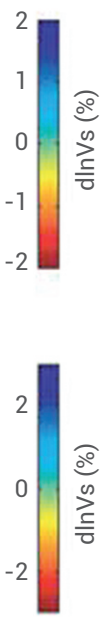

ङ

吾

4. Cartes de vitesse des ondes sismiques de cisaillement dans le manteau aux profondeurs de 100, 600, 1000 et 2800 km. L'échelle de couleur donne les variations relatives de vitesse par rapport à la moyenne à la profondeur donnée, interprétées en termes de température. En rouge, vitesse plus lente et température plus élevée ; en bleu, vitesse plus élevée et température plus basse. (D'après A.M. Dziewonski et al., Earth Planet. Sci. Lett. 299 (2010) 69-79).

\section{$>>$}

très grandes longueurs d'ondes. Deux structures de vitesses sismiques lentes, certainement chaudes, mais peut-être aussi de composition différente, sont à peu près centrées sur l'équateur, sous le Pacifique et sous l'Afrique. On les appelle parfois les «super-panaches ». Elles sont entourées de bandes de vitesses plus rapides, qui correspondraient au "cimetière de plaques ": endroits où les plaques recyclées dans le manteau se sont accumulées à la base de celui-ci au cours des temps géologiques. La nature et le rôle de ces structures à grande échelle font encore actuellement l'objet de débats et de recherches très actives.

\section{Sismogrammes numériques de la Terre}

Jusqu'à présent, l'essentiel de la tomographie était basée sur des hypothèses théoriques très simples sur la propagation des ondes dans une Terre « à trois dimensions ", comme la "théorie des rais ${ }^{(c)}$ " (équivalent des lois de Fresnel en optique) et la théorie des modes propres de la Terre (un sismogramme complet est décrit comme la somme de modes propres). Ces approximations supposent que les structures ne s'écartent que faiblement de la symétrie sphérique et ne permettent pas de prendre en compte des variations latérales rapides de structure. Nous nous sommes maintenant affranchis de ce problème par le calcul très précis du champ des ondes (c'est-à-dire des déplacements de matière) par la méthode rique d'éléments finis avec des polynômes d'ordre élevé, qui s'adapte bien à la géométrie de la sphère et aux calculs parallèles. Ceci est rendu possible par le développement rapide de la puissance des ordinateurs. On peut donc exploiter plus à fond et de manière plus précise l'information contenue dans les sismogrammes.

Nous avons ainsi gagné en précision, en particulier pour détecter les zones de vitesses sismiques lentes de faible étendue. En effet, lorsque les ondes émises par un séisme se propagent dans une zone contenant une telle "structure lente ", les ondes qui arrivent en premier à la station de détection sont celles qui ont contourné cette structure. La structure lente est alors " cachée " par ce phénomène qu'on appelle "wavefront healing". Pour décrire de façon précise les zones lentes, il faut faire appel à une très large bande de fréquences et analyser des sismogrammes complets. Les deux exemples suivants montrent des applications de ces techniques, actuellement en plein développement.

Le premier exemple est l'observation des trajets entre un séisme de l'ouest du Pacifique et le réseau très dense nordaméricain de stations sismiques (fig. 5a). des éléments spectraux, une méthode numé-
On étudie les ondes diffractées à la limite noyau-manteau sur ces trajets, et notamment sur la frontière nord du "super-panache pacifique " observé en rouge sur la figure $4 \mathrm{~d}$. Dans un modèle tomographique lisse (continu), on ne trouve pas de variation de la forme de l'onde de cisaillement diffractée le long de la limite sismique entre le noyau et le manteau en fonction de la position de la station (fig. $5 \mathrm{~b}$ droite), sauf une augmentation progressive du temps d'arrivée pour les trajets qui passent dans le super-panache. Les formes d'onde observées (fig. $5 b$ gauche) montrent toutefois un champ d'onde beaucoup plus complexe, avec des variations d'amplitude considérables : l'amplitude des ondes diffractées diminue fortement à la traversée de la frontière des super-panaches, et on voit apparaitre des ondes secondaires qui ont interagi avec une structure " anormale " localisée près de la limite noyau-manteau (fig. 5c). Ceci nous a amenés à proposer la présence d'une zone très particulière à la base du manteau : elle peut se modéliser au premier ordre par un cylindre d'une hauteur de $25 \mathrm{~km}$ et d'un diamètre d'environ $800 \mathrm{~km}$ (fig. 5d). La réduction de vitesse des ondes de cisaillement y serait de $20 \%$, ce qui est considérable puisque les variations latérales de vitesse obtenues par tomographie sismique à cette profondeur (fig. 4) ne dépassent pas 3 à 4\% en général.

Le deuxième exemple concerne des résultats récents dans le manteau supérieur, les premiers obtenus par l'application des techniques numériques à la tomographie. Un phénomène très intéressant mis en évidence par les méthodes numériques est l'existence de "zones lentes ", à $250 \mathrm{~km}$ de profondeur, dont la structure est particulière (S. French et al., Science 342 (2013) 227-230). Aussi bien dans l'océan Pacifique que dans l'Antarctique, l'océan Atlantique et l'océan Indien, ces zones où la vitesse de propagation des ondes $\mathrm{S}$ est ralentie s'alignent dans la direction du mouvement des plaques (e.g. fig. 6a). Sur les coupes verticales à travers le Pacifique (fig. 6b) apparaissent des structures très singulières organisées de manière quasi périodique, avec une longueur d'onde de l'ordre de $2000 \mathrm{~km}$ entre les zones plus lentes, qui persistent jusqu'à des profondeurs de 250 à $300 \mathrm{~km}$, et des zones intermédiaires plus rapides. Serait-ce la première mise en évidence dans le manteau terrestre des cellules de convection de Richter évoquées plus haut? 

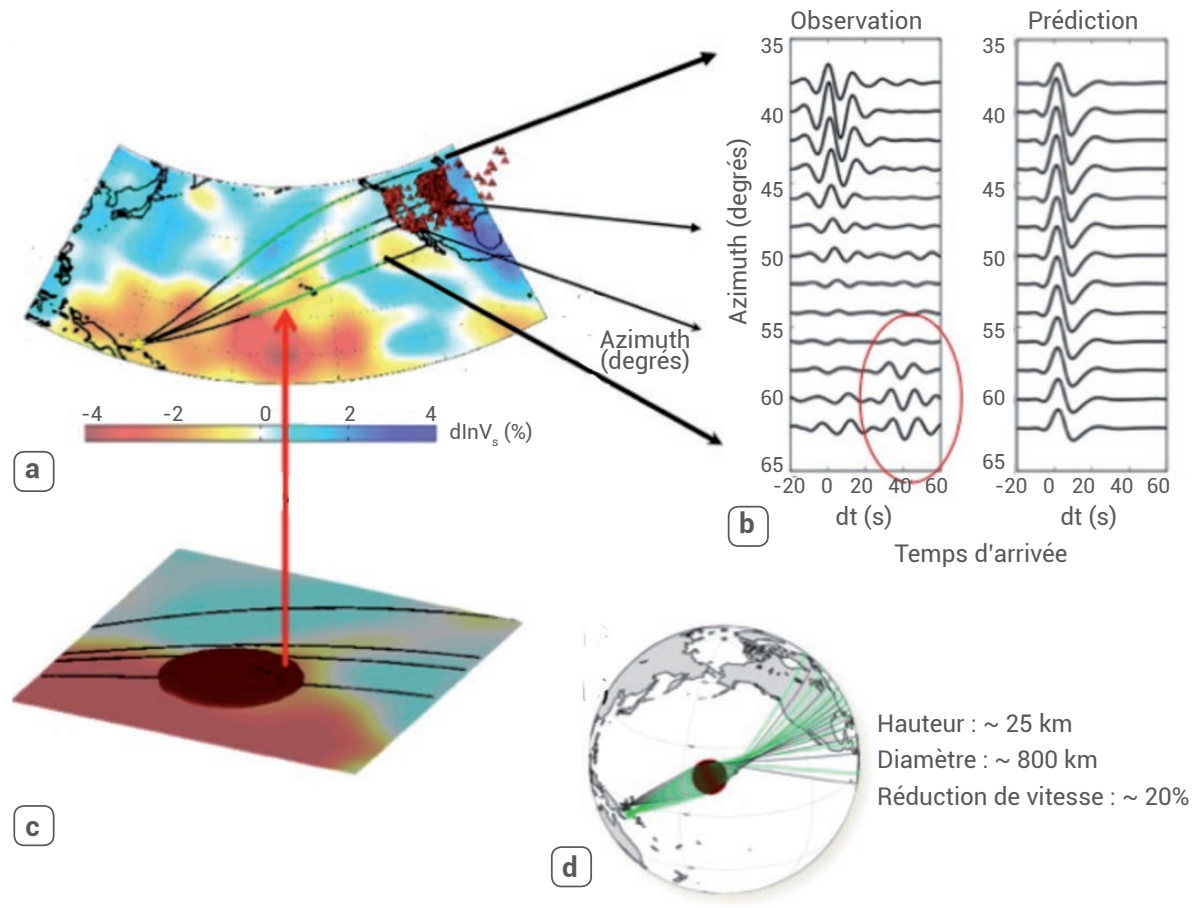

Hauteur : $25 \mathrm{~km}$

Diamètre : $800 \mathrm{~km}$

Réduction de vitesse : 20\%

c

d

5. Détection d'une zone de vitesse « ultralente » des ondes $\mathrm{S}$ à la base du manteau terrestre.

(a) Distribution des vitesses de cisaillement à $2800 \mathrm{~km}$ de profondeur sous l'océan Pacifique dans le modèle tomographique 3D SAW24816 de C. Mégnin et B. Romanowicz (Geophys. J. Int. 143 (2000) 709-728). Sont aussi indiquées les projections des trajets de quelques ondes $\mathrm{S}$ diffractées entre un séisme de l'ouest du Pacifique et les stations du réseau USArray aux USA. En vert, la portion de trajet qui échantillonne les derniers $300 \mathrm{~km}$ à la base du manteau.

(b) On compare les formes d'ondes observées (à gauche) avec les formes d'ondes calculées (à droite) dans le modèle tomographique montré en (a). L'ellipse rouge entoure les ondes secondaires.

(c) Représentation de la zone de vitesse " ultralente " des ondes S permettant d'expliquer la complexité des formes d'onde observées en (b).

(d) Cette zone agit comme une lentille, défléchissant les trajets des ondes. (s. Cottaar et B. Romanowicz, Earth Planet. Sci. Lett. 355-356 (2012) 213-222).

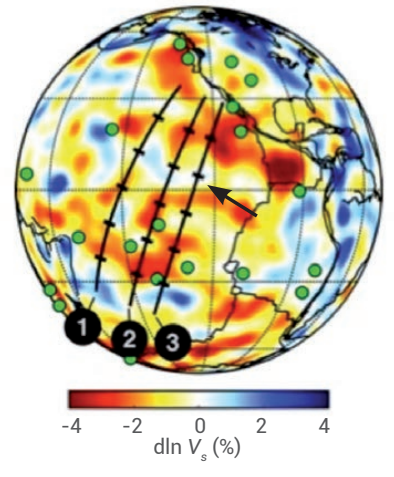

Perturbation de vitesse par rapport à la moyenne

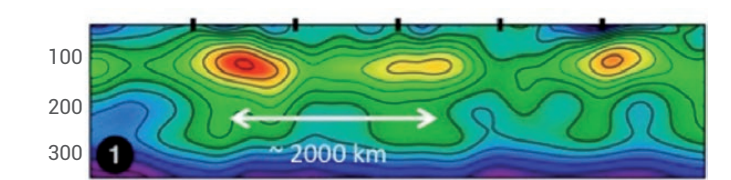

a
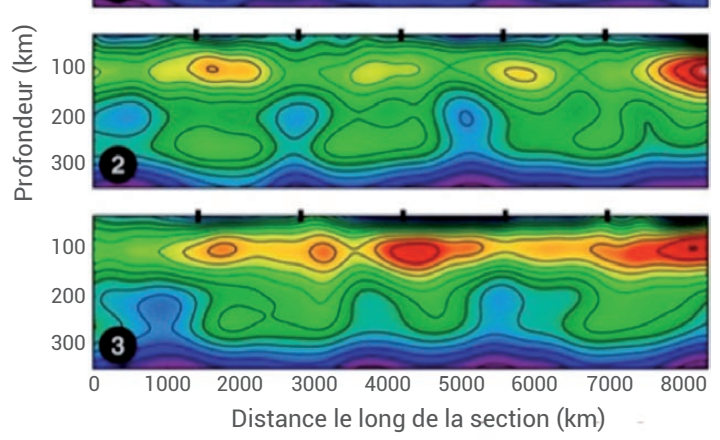

b

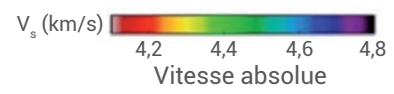

6. Zones "lentes".

(a) Variations latérales relatives de la vitesse des ondes $S$ à $250 \mathrm{~km}$ de profondeur dans le modèle SEMum2, exprimées par rapport à la moyenne globale à cette profondeur. La flèche noire indique la direction absolue du mouvement de la plaque Pacifique. Les zones de couleur rouge, où la vitesse des ondes $\mathrm{S}$ est ralentie, sont parallèles à cette flèche.

(b) Coupes verticales selon les profils 1, 2 et 3 indiqués en noir sur la carte en (a). Noter que dans ces coupes la vitesse est représentée en unités absolues $(\mathrm{km} / \mathrm{s})$.

\section{Conclusion}

Jusqu'aux années 1980, notre discipline a travaillé avec des modèles portant sur des valeurs moyennes de la structure sismique en fonction de la profondeur. Avec le développement des méthodes tomographiques, on a pu accéder aux hétérogénéités de grande échelle ; aujourd'hui les informations sont beaucoup plus précises, grâce à la puissance des ordinateurs appliquée au calcul numérique du champ des ondes. Dans le futur, nous pensons atteindre une encore meilleure résolution et donc accéder à des structures de plus en plus fines. L'espoir de la tomographie sismique repose sur des méthodes plus performantes de calcul du champ des ondes, mais aussi sur des réseaux transportables denses de stations sismiques, tel par exemple le réseau USArray $^{(\mathrm{d})}$ à travers le continent nordaméricain, qui est en train de porter ses fruits. Il serait utile de pouvoir étendre ce réseau à d'autres régions du globe, en particulier dans les océans. Ce n'est pas un problème technologique, mais de coût et de logistique.

B.R. remercie V. Pierron-Bohnes pour son travail remarquable de première rédaction à partir de la transcription de la conférence donnée au congrès SFP de Marseille.

(a) Principe et fonctionnement d'un sismomètre : on trouvera sur le site du Musée de sismologie et de la collection de géophysique de l'Université de Strasbourg toutes les informations et explications nécessaires : http://musee-sismologie.unistra.fr/ comprendre-les-seismes/notions-pour-petits-etgrands/les-sismometres/.

(b) La subduction (fig. 3b) provoque parfois une déformation extensive de la plaque supérieure, faisant naitre un bassin en arrière de l'arc volcanique principal. L'amincissement de la lithosphère au niveau de ce bassin d'arrière-arc peut favoriser l'apparition d'un volcanisme secondaire.

(c) Les rais sismiques sont les équivalents des rayons pour les ondes lumineuses : des trajectoires issues du foyer sismique et perpendiculaires en tout point aux fronts d'onde. Selon le principe de Fermat, le temps mis par un ébranlement pour aller d'un point à un autre est minimum le long des rais. Dans la Terre, la vitesse des ondes augmentant avec la profondeur, les rais sont courbes (fig. 1b).

(d) USArray est un réseau de 400 stations sismiques espacées de $70 \mathrm{~km}$ les unes des autres, en partie transportables, installé progressivement d'ouest en est entre 2004 et 2013 aux États-Unis. Ce réseau permet d'étudier la structure fine de la lithosphère sous le continent nord-américain.

\section{Pour en savoir plus}

La plupart des articles de B. Romanowicz sont accessibles sur :

http://seismo.berkeley.edu/wiki_br/Publications 